\title{
Reversible Severe Rhabdomyolysis Associated with Thyrotoxic Hypokalemic Periodic Paralysis
}

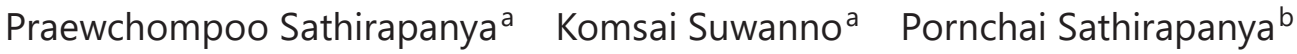 \\ aDepartment of Internal Medicine, Hat Yai Hospital, Hat Yai, Songkhla, Thailand; \\ bivision of Neurology, Department of Internal Medicine, Prince of Songkla University, \\ Hat Yai, Songkhla, Thailand
}

Keywords

Ion channel gene $\cdot$ Rhabdomyolysis · Endocrine gland · Electrolytes

\begin{abstract}
We report a case of reversible symptomatic rhabdomyolysis associated with thyrotoxic hypokalemic periodic paralysis. The patient had neither past medical nor family history of either disorder. The presenting neurological symptoms and signs, serum potassium, and creatine kinase levels returned to normal without specific treatment. Based on previous case reports, we attributed the combination of the disorders to a mutation of the calcium-gated channel (CACN) gene and its related encoded proteins.
\end{abstract}

\section{Introduction}

Hypokalemic periodic paralysis (HypoKPP) is a transient generalized muscular weakness caused by shifting of potassium into muscle cells resulting in hypokalemia. Rhabdomyolysis is rarely associated with HypoKPP, even in the cases of HypoKPP with severe hypokalemia. However, a few case reports suggested that mutations of the calcium-gated channel (CACN) gene and its related encoded proteins could cause rhabdomyolysis and HypoKPP concurrently $[1,2]$. Variant mutations of the CACN gene have been reported in association with a spectrum of muscular disorders of variable severity. Herein, we present a case of a first episode of transient rhabdomyolysis in combination with thyrotoxic HypoKPP and suggest that both conditions could manifest concomitantly based on the mutations of the CACN gene. 


\section{Case Report}

A 23-year-old male building painter had had mild myalgia and weakness on both of his thigh muscles, which did not disturb his daily career work, for few days before he fell from a 3-m-high scaffolding without significant bodily injuries. After presentation to a local hospital, severe myalgia developed on both of his thighs. The neurological examination revealed only mild and symmetrical muscle weakness of grade $4+/ 5$ on both arms and thighs. Traumatic rhabdomyolysis was the provisional diagnosis, and therefore serum creatine kinase (CK) and potassium were requested. The serum $\mathrm{CK}$ was $2,511 \mathrm{U} / \mathrm{L}$, while the serum potassium was 1.8 $\mathrm{mmol} / \mathrm{L}$. He was transferred to our hospital for further treatment. On arrival at our center $3 \mathrm{~h}$ after the first evaluation, the muscle weakness completely resolved, and the neurological examination was absolutely normal as well. At that time, a discoloration urine sample which fit with a diagnosis of myoglobinuria was collected, and the concomitant serum CK level was 2,433 U/L. However, the repeated serum potassium reported $5.2 \mathrm{mmol} / \mathrm{L}$. Thyrotoxicosis was additionally investigated and diagnosed by free triiodothyronine, free thyroxin, and thyrotropin-stimulating hormone of $18 \mathrm{pg} / \mathrm{dL}, 5.6 \mathrm{ng} / \mathrm{dL}$, and $0.003 \mu \mathrm{IU} / \mathrm{L}$, respectively. Subsequent serial tests of serum CK showed 1,751 and $507 \mathrm{U} / \mathrm{L}$ on the next 2 consecutive days without specific treatment. On the fifth admission day, the myalgia resolved completely as well as both serum CK and potassium levels normalized. The patient confirmed from the medical history review that his family history was clear of both rhabdomyolysis and thyrotoxic HypoKPP.

\section{Discussion}

We report a case of symptomatic rhabdomyolysis associated with short-lasting thyrotoxic HypoKPP. While HypoKPP has been commonly associated with thyrotoxicosis or euthyroid state, rhabdomyolysis has scarcely been reported in association with thyrotoxicosis or HypoKPP $[3,4]$. However, a recent case report suggested that mutations of the calcium-gated channel alpha 1 subunit (CACNA1S) gene and its 2 related encoded proteins, $\mathrm{Ca}_{\mathrm{v}} 1.1$ and ryanodine receptor 1 (RYR1), were associated with a group of phenotypic variants of muscular disorders including HypoKPP. The CACNA1S gene encodes $\mathrm{Ca}_{\mathrm{v}} 1.1$ which forms a calcium-gated channel to act as a sensor for muscle excitation-contraction coupling. $\mathrm{Ca}_{\mathrm{v}} 1.1$ then activates RYR1 to induce the release of calcium from the sarcoplasmic reticulum. The dominant mutations of the CACNA1S gene were associated with HypoKPP, malignant hyperthermia, and congenital myopathy, while mutations of RYR1 caused rhabdomyolysis, malignant hyperthermia, and congenital myopathy [2]. The typical variants of the CACNA1S gene responsible for HypoKPP reported to date are p. Arg528His, p. Arg528Gly, and p. Arg528Cys. However, a case with the p. Arg528Leu variant with isolated rhabdomyolysis without clinical evidence of HypoKPP has been reported recently [2]. This supports the principle that the mutations of the specific gene, which is responsible for well-recognized muscular disorders, can present with variable phenotypes of muscular disorders. The concurrent appearance of HypoKPP and rhabdomyolysis in our case could be caused by another unique CACNA1S gene variant. Unfortunately, as genetic analysis is unavailable in our hospital, we were unable to elucidate the specific mutations of the CACNA1S and RYR1 gene in this case. In our literature review, we found a known case of HypoKPP who presented repeatedly with periodic muscular weakness, but with only one incident of concurrent rhabdomyolysis in the same time as severe hypokalemia [4]. In contrast, Anandan et al. [2] reported a case of the CACNA1S variant who presented with rhabdomyolysis and fluctuating asymptomatic hyper-CKemia, but no concurrent HypoKPP presented. Unlike the previously reported cases, our case presented with both of mild and transient muscle weakness of HypoKPP but dominantly severe symptomatic rhabdomyolysis.

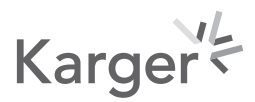




\section{Conclusion}

Similar to the earlier cases, our reported patient could be a case of muscular disorder caused by the previously unknown mutations of the CACNA1S gene and/or one or more of its related encoded proteins.

\section{Acknowledgment}

The authors thank David Patterson of the International Affairs Unit of Faculty of Medicine, Prince of Songkla University, for English language editing of the manuscript.

\section{Statement of Ethics}

This case report was reviewed and approved by the Institutional Ethics Committee, Hat Yai Hospital (Registration No. 44/2563). The authors confirmed that they strictly followed the guideline of 1964 Declaration of Helsinki and related good clinical practices in writing this case report. The patient's identifiable information and identity was completely anonymized. The patient gave written consent to report and publish this case report. The authors accept the journal's regulations on the issues involved in ethical publication and affirmed that this report was consistent with those regulations.

\section{Conflict of Interest Statement}

The authors declare that they have no conflicts of interest to disclose.

\section{Funding Sources}

No grant or support was received.

\section{Author Contributions}

\begin{tabular}{ll}
\hline Name & Contribution \\
\hline Praewchompoo Sathirapanya (author) & $\begin{array}{l}\text { Acquisition of patient's clinical information and } \\
\text { clearance. Cowrote the first draft of the manuscript. } \\
\text { Approval of the final manuscript } \\
\text { Komsai Suwanno (author) }\end{array}$ \\
$\begin{array}{l}\text { Acquisition of patient's clinical information and } \\
\text { clearance. Cowrote the first draft of the manuscript. } \\
\text { Approval of the final manuscript }\end{array}$ \\
Pornchai Sathirapanya* (corresponding author) & $\begin{array}{l}\text { Conceptualization of the intellectual content. Data } \\
\text { interpretation. Outlined the manuscript content. } \\
\text { Editing and approval of the final version of the } \\
\text { manuscript before submission }\end{array}$ \\
\hline
\end{tabular}




\section{References}

1 Neame MT, Wright D, Chandrasekaran S. Persisting fatigue and myalgia as the presenting features in a case of hypokalaemic periodic paralysis. BMJ Case Rep. 2017 Aug 10;2017:bcr2017219991.

2 Anandan C, Cipriani MA, Laughlin RS, Niu Z, Milone M. Rhabdomyolysis and fluctuating asymptomatic hyperCKemia associated with CACNA1S variant. Eur J Neurol. 2018 Feb;25(2):417-9.

3 Summachiwakij S, Sachmechi I. Rhabdomyolysis induced by nonstrenuous exercise in a patient with graves' disease. Case Rep Endocrinol. 2014;2014:286450.

4 Jung YL, Kang JY. Rhabdomyolysis following severe hypokalemia caused by familial hypokalemic periodic paralysis. World J Clin Cases. 2017 Feb 16;5(2):56-60. 